\title{
Viewing the healthcare system through a deaf
} lens

\section{Sherrie Beaver $r^{a, c}$ and Breda Carty ${ }^{b}$}

a School of Health and Social Development, Deakin University, Melbourne, VIC, Australia

b School of Education, Macquarie University, Sydney, NSW, Australia

c Corresponding author: sherrie@isigniwander.com

\section{Article history}

Publication date: 2 December 2021

Citation: Beaver S, Carty B. Viewing the healthcare system through a deaf lens. Public Health Res Pract. 2021;31(5):e3152127. https://doi. org/10.17061/phrp3152127

\section{Key points}

- The healthcare system has built-in barriers for deaf people, such as inadequate access to Auslan (Australian Sign Language) interpreters

- Research about deaf people and their health should be conducted with the participation of deaf people

- The coronavirus disease 2019 (COVID-19) pandemic offers valuable lessons for communicating with deaf and hard of hearing people, particularly when people are wearing face masks

\section{Abstract}

This perspective paper examines some common barriers to effective participation in the healthcare system experienced by deaf people. The presentation of research and policy is considered from a deaf perspective and in the context of the challenges and opportunities raised by responses to the coronavirus disease 2019 (COVID-19) pandemic.

\section{Introduction}

We are writing at a time of heightened social awareness of the importance of effective public healthcare, including the key role of accessible communication. A global pandemic presents unique challenges in addressing barriers to shared understanding and responses - deaf and hard of hearing people's experiences navigating health systems exemplify many of the issues public health practitioners are now grappling with.

We write as deaf women whose preferred language is Auslan (Australian Sign Language) but acknowledge the great diversity among deaf and hard of hearing people. The terms 'deaf', 'Deaf', 'hard of hearing' and 'hearing impaired' have shifting meanings in the literature. In this paper, we use the lower case 'deaf' - or sometimes 'deaf and hard of hearing' - to refer to all people with hearing loss. As many as one in six Australians experience deafness or hearing loss. ${ }^{1}$ Most have age-related hearing loss; others are deaf from birth or early childhood. There is wide variation in age of onset, educational background, communication preferences and use of hearing devices. Whatever our background, all too often we experience the healthcare system as a series of barriers, many of which relate to the assumption that as deaf people we have some inherent health deficit. How many times have we been to a doctor for a sore throat and had to field a series of questions about how old we were when we became deaf and whether we have considered a cochlear implant or other treatment?

Such experiences exemplify the contrast between medical models of disability - which focus on diagnosis, prevention and treatment, and perceive the disability as residing in the individual; and social models - which focus on systemic barriers, such as inaccessible communication, that impose disabling limits on individuals and groups. ${ }^{2}$ 


\section{Barriers in the healthcare system experienced by deaf people}

Health literacy is one of the most common barriers deaf people face in the healthcare system. Information about medical conditions and illnesses is usually available in English and other languages in printed form. However, people who are deaf from birth or early childhood may experience lower levels of literacy because of educational disadvantage. Evidence suggests that deaf people graduate from secondary school with average reading levels of grade $4-7 .{ }^{3}$ Lack of access to incidental information such as family conversations may also leave deaf people with a limited fund of basic information. These people are at risk of receiving inadequate information to manage and make informed choices about their health and, consequently, they experience reduced autonomy. ${ }^{4} \mathrm{~A}$ study commissioned by the Australian Government found that individual doctors acknowledged a high risk of misunderstanding and health mismanagement if an Auslan interpreter was not present during complex medical appointments with deaf patients. $^{5}$

Access to interpreting in the healthcare system is another common barrier experienced by deaf people who use Auslan or other signed languages. Healthcare staff often lack understanding of availability or booking processes for Auslan interpreters, which can result in deaf people being burdened with self-advocacy to be able to access the healthcare system. This can cause unneeded distress for patients and their families. Where there is no Auslan interpreter present, deaf people are at high risk of misunderstanding and/or receiving incorrect information to manage their health, particularly during complex medical appointments. ${ }^{5}$ When a deaf patient does not receive clear communication about their healthcare, healthcare staff may experience difficulty with negotiating proper consent, which can have serious consequences for deaf patients' autonomy.

Family members and friends of deaf people are regularly asked by healthcare providers to interpret for deaf patients' medical appointments. Children of deaf adults, in particular, often carry this expectation. This 'language brokering' occurs when children take on the role of interpreting, translating and advocating for their parents. ${ }^{6}$ However, peak body Deaf Australia argues that family and friends are not equipped to interpret for deaf people during medical appointments and that this practice risks leaving patients and their family members or friends traumatised by the experience. Rather than interpreting, family members and friends, including children of deaf adults, should instead be available to provide practical or emotional support to the deaf person during healthcare appointments. ${ }^{7}$

Deaf people encounter varying attitudes from healthcare professionals. Some are enlightened but others make assumptions about deaf people's intelligence and character based on their ability to hear or to use spoken language - an attitude often referred to as 'audism'. ${ }^{8}$ Laur states that deaf people often experience barriers because of health professionals' attitudes, lack of understanding and inadequate training for meeting the needs of deaf patients. Deaf people can experience better satisfaction when they are shown empathy and understanding during healthcare appointments and during follow-up appointments and treatment. ${ }^{9}$

\section{Research and its interpretation and reporting}

It can be a dispiriting experience for deaf people to read some of the research about our health status and how it is perceived by clinicians. However, to read nuanced research with examples from lived experience and pertinent recommendations, especially when deaf researchers have been involved throughout the research and reporting, is sobering but useful. ${ }^{3}$ Too much research presents blanket assertions that deafness per se is a risk factor for poor physical and mental health. ${ }^{10}$ It can be frustrating when research does not clearly tease out the elements creating risk for deaf people, such as poor education, limited communication and inadequate access to social participation and public services. It would be helpful if it was made clearer that these experiences are not inevitable. Many deaf people can and do acquire language (signed, spoken or both) in age-appropriate ways, receive equitable education, perform satisfying and useful work, enjoy enriching relationships and social opportunities, advocate for and make use of supports enabling them to participate in social and cultural life and enjoy good health. This needs to be acknowledged and celebrated.

The cumulative effect of some of the language used about deaf people in health-related research and policy is also alienating. Various forms of deafness are referred to as 'disorders' or even 'diseases', deaf babies are reported to 'fail' their Auditory Brainstem Response tests ${ }^{11}$, and deafness and hearing loss are routinely described as 'burdens' -usually on a national or global scale. ${ }^{12,13}$ Apart from the casually negative language, such descriptions also suggest powerfully that deaf people have not been involved in the research or its presentation. We are 'othered' by this language.

\section{What can we learn from the COVID-19 pandemic?}

The coronavirus disease 2019 (COVID-19) pandemic has provided a salutary case study of a public health crisis in which accessible information is critical for the whole community. If sections of the public are not informed about symptoms, treatment and containment measures 
in such a rapidly evolving pandemic, it affects everyone's health and safety.

The requirement to wear face masks has provided immediate communication challenges for deaf people. Half-hearted 'exemptions' advising people to remove their masks to communicate with a deaf person have been inconsistently observed, and transparent masks or shields are not yet a viable solution. As mask-wearing is likely to be a feature of public life for some time, better solutions need to be found, and more robust access provisions such as interpreting and real-time captioning need to be supported.

Sign language interpreters and real-time captioning for public health announcements have been visible during the pandemic in many countries, although access is still incomplete and inconsistent. Panko et al. found that deaf adults in the US were able to identify symptoms of COVID-19 equally as well as hearing people, despite being much more likely to report problems accessing relevant information. ${ }^{14}$ Murray reported that deaf community organisations in many countries were preparing and disseminating information independently within their communities, allowing for targeted delivery taking into account the language and cultural needs of deaf people. ${ }^{15}$

The pandemic has also led to greater flexibility in provision of health services, with a rise in e-health delivery. Although this has yet to be made accessible to most deaf consumers, it offers possibilities for more tailored health services, for example to connect deaf patients with healthcare providers who have deaf awareness and/or Auslan skills but are in a different location.

\section{Conclusion}

The healthcare system not only manages and treats illness; it often functions as a gateway for access to other social services or supports. The interconnectedness of our systems can add an extra layer of difficulty for those whose access is tenuous. The healthcare system is based on a medical model of disability, where deafness is usually seen as a condition needing to be fixed. Deaf people are more likely to operate within a social model of disability where we are acknowledged as whole individuals facing systemic communication barriers. This disconnect regularly causes access issues for deaf people seeking healthcare services. As highlighted clearly by the COVID-19 pandemic, improving the accessibility and awareness of our healthcare system could have far-reaching social and health benefits for deaf people.

\section{Peer review and provenance}

\section{Competing interests}

None declared.

\section{Author contributions}

Each author contributed equally to the preparation and review of the manuscript.

\section{References}

1. Access Economics Pty Ltd. Listen Hear! The economic impact and cost of hearing loss in Australia. Victoria: Access Economics; 2006 [cited 2021 Jul 29]. Available from: hearnet.org.au/wp-content/uploads/2015/10/ ListenHearFinal.pdf

2. Berger RJ, Lorenz, LS. Disability and qualitative research. In: Berger RJ, Lorenz LS, editors. Disability and qualitative enquiry: methods for rethinking an ableist world. New York: Routledge; 2015. p. 1-10.

3. Vermeulen AM, van Bon W, Schreuder R, Knoors H, Snik A. Reading comprehension of deaf children with cochlear implants. J Deaf Stud Deaf Educ. 2007;12(3):283-302.

4. Witko J, Boyles P, Smiler K, McKee R. Deaf New Zealand Sign Language users' access to healthcare. N Z Med J. 2017;130(1466):53-61.

5. Napier J, Kidd MR. English literacy as a barrier to healthcare information for deaf people who use Auslan. Aust Fam Physician. 2013;42(12):896-9.

6. Moroe N, De Andrade V. "We were our parents' ears and mouths:" Reflecting on the language-brokering experiences of hearing children born to deaf-parents. S Afr J Child Health. 2018;12(2b):75.

7. Deaf Australia. Accessible services for deaf people who use Auslan in hospitals and health services Prepared for Department of Health. 2021. Available from authors.

8. Bauman H-DL. Audism: Exploring the metaphysics of oppression. J Deaf Stud Deaf Educ. 2004;9(2):239-46.

9. Laur A. Healthcare access for deaf patients - the legal and ethical perspectives. Med Leg J. 2017;86(1):36-41.

10. Abrams $\mathrm{H}$. Hearing loss and associated comorbidities: what do we know? Kansas City: The Hearing Review; 2017 [cited 2021 Nov 16]. Available from: hearingreview. com/hearing-loss/hearing-loss-prevention/risk-factors/ hearing-loss-associated-comorbidities-know

11. Holster IL, Hoeve LJ, Wieringa MH, Willis-Lorrier RMS, de Gier HHW. Evaluation of hearing loss after failed neonatal hearing screening. J Pediatr. 2009;155(5):64650.

12. Wilson BS, Tucci DL. Addressing the global burden of hearing loss. Lancet. 2021;397(10278):945-7.

Externally peer reviewed, invited. 
13. Graydon K, Waterworth C, Miller H, Gunasekera H. Global burden of hearing impairment and ear disease. J Laryngol Otol. 2019;133(1):18-25.

14. Panko TL, Contreras J, Postl D, Mussallem A, Champlin S, Paasche-Orlow MK, et al. The deaf community's experiences navigating COVID-19 pandemic information. Health Lit Res Pract. 2021;5(2):e162-70.
15. Murray JJ. Improving signed language and communication accessibility during COVID-19 pandemic. The Hearing Journal. 2020 May 26; online ahead of print.

\section{Copyright: (c) (9)}

(C) 2021 Beaver and Carty. This article is licensed under the Creative Commons Attribution-NonCommercial-ShareAlike 4.0 International Licence, which allows others to redistribute, adapt and share this work non-commercially provided they attribute the work and any adapted version of it is distributed under the same Creative Commons licence terms. See: www.creativecommons.org/licenses/by-nc-sa/4.0/ 